\title{
ON A PAIRING BETWEEN SYMMETRIC POWER MODULES
}

\author{
FRAZER JARVIS \\ School of Mathematics and Statistics, University of Sheffield, Hicks Building, Sheffield S3 7RH, UK \\ e-mail:a.f.jarvis@shef.ac.uk
}

(Received 29 February 2012; accepted 15 March 2012; first published online 2 August 2012)

\begin{abstract}
We prove, using purely combinatorial methods, that there is a pairing $\operatorname{Sym}^{a} \mathbb{Q}^{2} \times \operatorname{Sym}^{a} \mathbb{Q}^{2} \longrightarrow \mathbb{Q}$
\end{abstract}

with an $M_{2}(\mathbb{Q})$-equivariance property.

2000 American Mathematics Subject Classification. 11F33, 11F41, 11F80, 11G18.

1. Introduction. In associating the Galois representations to modular eigenforms of weight $k$, one considers étale cohomology of modular curves with coefficients that are essentially $\ell$-adic sheaves $\operatorname{Sym}^{k-2} \mathbb{Q}_{\ell}^{2}$. In order to prove properties of the Galois representations, we need to know as much as possible about these cohomology groups. It was asserted by Taylor [4, p. 270] that there is an explicit pairing $\langle, \quad\rangle: \operatorname{Sym}^{a} R^{2} \times$ $\operatorname{Sym}^{a} R^{2} \longrightarrow R$ for any ring $R$ with the property that $\langle x \alpha, y \alpha\rangle=\operatorname{det}(\alpha)^{a}\langle x, y\rangle$, where $\alpha \in M_{2}(R)$ has an induced right action on the symmetric power module. This was used there (and elsewhere) to give an explicit Poincaré duality on the étale cohomology groups, leading to a clearer understanding of the Galois representations.

However, as Kevin Buzzard pointed out to the author, the pairing defined by Taylor [4] does not actually satisfy the asserted property. In this note, we alter Taylor's definition slightly, and show, using entirely elementary combinatorial methods, that the desired property holds, at least if the ring has characteristic 0 . Jordan and Livné [2, Corollary 2.16] also seem to prove the existence of such a pairing, using properties of quaternion algebras, but the proof we give is rather more explicit, as well as elementary.

This result repairs the gap in [4] so that the results there are now valid for characteristic 0 and also all but finitely many finite characteristics. The result was used in the PhD thesis of the author's student Crane [1], where level raising was considered for the Galois representations over totally real fields; this level raising is also treated in a recent work of Kisin [3].

2. The main theorem. For the moment, we will let $R$ denote a $\mathbb{Q}$-algebra so that every number is invertible. We let $R^{2}$ have standard basis $\left\{e_{1}, e_{2}\right\}$. Then there is an obvious action of a matrix $\alpha=\left(\begin{array}{ll}\alpha_{11} & \alpha_{12} \\ \alpha_{21} & \alpha_{22}\end{array}\right) \in M_{2}(R)$ on $R^{2}$, which we write on the right (as in [4]); the basis element $e_{1}=\left(\begin{array}{ll}1 & 0\end{array}\right)$ is mapped to $\left(\alpha_{11} \alpha_{12}\right)=\alpha_{11} e_{1}+\alpha_{12} e_{2}$, and similarly $e_{2}$ is mapped to $\alpha_{21} e_{1}+\alpha_{22} e_{2}$. This action induces an action on $\operatorname{Sym}^{a} R^{2}$, where we take the standard basis $\left\{e_{1}^{\otimes a}, e_{1}^{\otimes a-1} \otimes e_{2}, \ldots, e_{2}^{\otimes a}\right\}$; the basis element $e_{1}^{\otimes i} \otimes e_{2}^{\otimes a-i}$ is mapped to $\left(\alpha_{11} e_{1}+\alpha_{12} e_{2}\right)^{\otimes i} \otimes\left(\alpha_{21} e_{1}+\alpha_{22} e_{2}\right)^{\otimes a-i}$, and we extend this linearly to all elements of $\mathrm{Sym}^{a} R^{2}$. (One can think of $\operatorname{Sym}^{a} R^{2}$ as homogeneous polynomials of degree $a$, spanned by $x^{a}, x^{a-1} y, \ldots, y^{a}$.) 
Define the matrix $W$ to be the $(a+1) \times(a+1)$-matrix, where we index the rows and columns from $0, \ldots, a$, by

$$
W_{i j}=\left\{\begin{array}{ll}
0, & \text { if } i+j \neq a \\
(-1)^{i}\left(\begin{array}{l}
a \\
i
\end{array}\right)^{-1}, & \text { if } i+j=a
\end{array} .\right.
$$

Define the pairing

$$
\begin{aligned}
\operatorname{Sym}^{a} R^{2} \times \operatorname{Sym}^{a} R^{2} & \longrightarrow R \\
(x, y) & \mapsto x W y^{t},
\end{aligned}
$$

where, as above, we think of elements as row vectors with respect to the standard basis. With this notation, we claim that this pairing satisfies the required property.

THEOREM 2.1. Let $x, y \in \operatorname{Sym}^{a} R^{2}$. Then for any matrix $\alpha=\left(\begin{array}{ll}\alpha_{11} & \alpha_{12} \\ \alpha_{21} & \alpha_{22}\end{array}\right) \in M_{2}(R)$,

$$
\langle x \alpha, y \alpha\rangle=\operatorname{det}(\alpha)^{a}\langle x, y\rangle .
$$

Proof. Let us write $A$ for the $(a+1) \times(a+1)$-matrix giving the action of $\alpha$ on $\operatorname{Sym}^{a} R^{2}$ with respect to the standard basis.

A short, elementary calculation shows that $A_{k l}$ is given by

$$
\sum_{t=0}^{a}\left(\begin{array}{c}
a-k \\
t
\end{array}\right)\left(\begin{array}{c}
k \\
l-t
\end{array}\right) \alpha_{11}^{a-k-t} \alpha_{12}^{t} \alpha_{21}^{k+t-l} \alpha_{22}^{l-t}
$$

where we again index rows and columns from 0 to $a$. Then the claim is equivalent to

$$
A W A^{t}=d^{a} W
$$

where $d=\operatorname{det} \alpha=\alpha_{11} \alpha_{22}-\alpha_{12} \alpha_{21}$.

It is easy to see, however, that this equality is equivalent to the equality $A^{t} W^{-1} A=$ $d^{a} W^{-1}$. It therefore suffices to prove something similar for the transpose matrix. The entries of $W^{-1}$ are just like those of $W$ except that the $k l$ th entry is $(-1)^{a-k}\left(\begin{array}{l}a \\ k\end{array}\right)$ if $k+l=a$.

We need to see that if $i+j \neq a$, then the $i j$ th entry of $A^{t} W^{-1} A$ vanishes, and if $i+j=a$, then the $i j$ th entry is $d^{a}(-1)^{a-i}\left(\begin{array}{c}a \\ i\end{array}\right)$.

Using the expression for $A_{k l}$, a calculation gives the $i$ th entry of $A^{t} W^{-1} A$ to be

$$
\sum_{r=0}^{a} \alpha_{11}^{a-r} \alpha_{12}^{r} \alpha_{21}^{a+r-i-j} \alpha_{22}^{i+j-r}\left[\sum_{m=0}^{a}(-1)^{a-m}\left(\begin{array}{c}
a \\
m
\end{array}\right) \sum_{s=0}^{a}\left(\begin{array}{c}
a-m \\
s
\end{array}\right)\left(\begin{array}{c}
m \\
i-s
\end{array}\right)\left(\begin{array}{c}
m \\
r-s
\end{array}\right)\left(\begin{array}{c}
a-m \\
j+s-r
\end{array}\right)\right] .
$$

Write the term $\alpha_{11}^{a-r} \alpha_{12}^{r} \alpha_{21}^{a+r-i-j} \alpha_{22}^{i+j-r}$ as $\alpha_{11}^{a} \alpha_{21}^{a-i-j} \alpha_{22}^{i+j} z^{r}$, where $z$ is $\frac{\alpha_{12} \alpha_{21}}{\alpha_{11} \alpha_{22}}$.

Then our sum can be rewritten as

$$
\alpha_{11}^{a} \alpha_{21}^{a-i-j} \alpha_{22}^{i+j} \sum_{m=0}^{a}(-1)^{a-m}\left(\begin{array}{c}
a \\
m
\end{array}\right) \sum_{r=0}^{a} \sum_{s=0}^{a} z^{r}\left(\begin{array}{c}
a-m \\
s
\end{array}\right)\left(\begin{array}{c}
m \\
i-s
\end{array}\right)\left(\begin{array}{c}
m \\
r-s
\end{array}\right)\left(\begin{array}{c}
a-m \\
j+s-r
\end{array}\right),
$$


or, writing $t=r-s$,

$$
\alpha_{11}^{a} \alpha_{21}^{a-i-j} \alpha_{22}^{i+j} \sum_{m=0}^{a}(-1)^{a-m}\left(\begin{array}{c}
a \\
m
\end{array}\right) \sum_{t=0}^{a} \sum_{s=0}^{a} z^{s+t}\left(\begin{array}{c}
a-m \\
s
\end{array}\right)\left(\begin{array}{c}
m \\
i-s
\end{array}\right)\left(\begin{array}{c}
m \\
t
\end{array}\right)\left(\begin{array}{c}
a-m \\
j-t
\end{array}\right) .
$$

It is easy to see that the inner double sum is just the coefficient of $x^{i} y^{j}$ in the product $(1+x z)^{a-m}(1+x)^{m}(1+y z)^{m}(1+y)^{a-m}$. Therefore the sum

$$
\sum_{m=0}^{a}(-1)^{a-m}\left(\begin{array}{c}
a \\
m
\end{array}\right) \sum_{t=0}^{a} \sum_{s=0}^{a} z^{s+t}\left(\begin{array}{c}
a-m \\
s
\end{array}\right)\left(\begin{array}{c}
m \\
i-s
\end{array}\right)\left(\begin{array}{c}
m \\
t
\end{array}\right)\left(\begin{array}{c}
a-m \\
j-t
\end{array}\right)
$$

is the coefficient of $x^{i} y^{j}$ in

$$
\begin{aligned}
& \sum_{m=0}^{a}(-1)^{a-m}\left(\begin{array}{c}
a \\
m
\end{array}\right)(1+x z)^{a-m}(1+x)^{m}(1+y z)^{m}(1+y)^{a-m} \\
& \quad=[(1+x)(1+y z)-(1+x z)(1+y)]^{a}
\end{aligned}
$$

by the binomial formula. But

$$
(1+x)(1+y z)-(1+x z)(1+y)=(1-z)(x-y),
$$

so the coefficient of $x^{i} y^{j}$ in $[(1+x)(1+y z)-(1+x z)(1+y)]^{a}$ is the same as the coefficient of $x^{i} y^{j}$ in $(1-z)^{a}(x-y)^{a}$. But clearly there are no terms except in degree $a$, so unless $i+j=a$, the sum vanishes, as required.

If $i+j=a$, the sum

$$
\alpha_{11}^{a} \alpha_{21}^{a-i-j} \alpha_{22}^{i+j} \sum_{m=0}^{a}(-1)^{a-m}\left(\begin{array}{c}
a \\
m
\end{array}\right) \sum_{t=0}^{a} \sum_{s=0}^{a} z^{s+t}\left(\begin{array}{c}
a-m \\
s
\end{array}\right)\left(\begin{array}{c}
m \\
i-s
\end{array}\right)\left(\begin{array}{c}
m \\
t
\end{array}\right)\left(\begin{array}{c}
a-m \\
j-t
\end{array}\right)
$$

becomes the coefficient of $x^{i} y^{j}$ in

$$
\alpha_{11}^{a} \alpha_{22}^{a}(1-z)^{a}(x-y)^{a} .
$$

But

$$
1-z=1-\frac{\alpha_{12} \alpha_{21}}{\alpha_{11} \alpha_{22}}=\frac{\alpha_{11} \alpha_{22}-\alpha_{12} \alpha_{21}}{\alpha_{11} \alpha_{22}}=\frac{d}{\alpha_{11} \alpha_{12}},
$$

so that $\alpha_{11}^{a} \alpha_{22}^{a}(1-z)^{a}$ is just $d^{a}$, and we need the coefficient of $x^{i} y^{j}$ in $d^{a}(x-y)^{a}$, namely $d^{a}(-1)^{a-i}\left(\begin{array}{c}a \\ i\end{array}\right)$, which is $d^{a}$ multiplied by the $\ddot{j}$ th entry of $W^{-1}$, exactly as required.

REMARK 2.2. We stated this as a result for $\mathbb{Q}$-algebras, for simplicity. We only require that the binomial coefficients $\left(\begin{array}{l}a \\ 0\end{array}\right),\left(\begin{array}{l}a \\ 1\end{array}\right), \ldots,\left(\begin{array}{l}a \\ a\end{array}\right)$ should be invertible in $R$. Since no prime greater than $a$ divides any of these binomial coefficients, we see that the result holds for any ring $R$ in which primes up to $a$ are invertible. In particular, the claim holds if $R$ is any field of characteristic greater than $a$. (We should remark that Taylor [4] uses this claim only for rings that have this property, and the results of his paper therefore remain valid.)

Now that we know that the identity for $A^{t} W^{-1} A$ holds, the equivalent result for $A W A^{t}$ implies the following identity for binomial coefficients: If the numbers $a, i, j$ 
and $r$ are given, with $0 \leq r \leq a$, then

$$
\sum_{m=0}^{a} \frac{(-1)^{m}}{\left(\begin{array}{l}
a \\
m
\end{array}\right)}\left[\sum_{s=0}^{a}\left(\begin{array}{c}
a-i \\
s
\end{array}\right)\left(\begin{array}{c}
i \\
m-s
\end{array}\right)\left(\begin{array}{c}
a-j \\
a+r-i-j-s
\end{array}\right)\left(\begin{array}{c}
j \\
i+j+s-r-m
\end{array}\right)\right],
$$

or, equivalently, its obvious rearrangement

$$
\sum_{s=0}^{a}\left(\begin{array}{c}
a-i \\
s
\end{array}\right)\left(\begin{array}{c}
a-j \\
a+r-i-j-s
\end{array}\right)\left[\sum_{m=0}^{a} \frac{(-1)^{m}}{\left(\begin{array}{c}
a \\
m
\end{array}\right)}\left(\begin{array}{c}
i \\
m-s
\end{array}\right)\left(\begin{array}{c}
j \\
i+j+s-r-m
\end{array}\right)\right],
$$

is equal to 0 if $i+j \neq a$, and if $i+j=a$, it is

$$
(-1)^{i+r} \frac{\left(\begin{array}{l}
a \\
r
\end{array}\right)}{\left(\begin{array}{c}
a \\
i
\end{array}\right)}=(-1)^{i+r} \frac{i ! j !}{r !(a-r) !} .
$$

This follows because this is a coefficient of one of the monomials in the $i j$ th entry of $A W A^{t}$.

\section{REFERENCES}

1. M. Crane, Raising the levels of Hilbert modular forms, PhD Thesis (University of Sheffield, Sheffield, UK, 2007).

2. B. Jordan and R. Livné, Integral Hodge theory and congruences between modular forms, Duke Math. J. 80 (1995), 419-484.

3. M. Kisin, Moduli of finite flat group schemes, and modularity, Ann. Math. 170 (2009), $1085-1180$.

4. R. Taylor, On Galois representations associated to Hilbert modular forms, Invent. Math. 98 (1989), 265-280. 